\title{
1. INTRODUCCIÓN Y METODOLOGÍA
}

La Ley General de Sanidad de 1986 (1) en su Capítulo III propone la plena integración de la atención de los problemas de salud mental en el sistema sanitario general, aportando los principios básicos en los que debe basarse esa atención:

- La atención se realizará en el ámbito comunitario: recursos asistenciales ambulatorios, hospitalización parcial y atención a domicilio.

- Se considerarán de modo especial la Psicogeriatría y la Psiquiatría Infantil.

- La hospitalización se realizará en Unidades de Psiquiatría de Hospitales Generales.

- Se desarrollarán servicios de rehabilitación y reinserción social en coordinación, cuando sea necesario, con Servicios Sociales.

La Ley adjudicó la responsabilidad de transformar y organizar la atención a la salud mental a las administraciones sanitarias de las Comunidades Autónomas (CCAA). Cada CCAA acometió el proceso de reforma de la atención a la salud mental con arreglo a su propia historia, y es por ello que, en cada una de ellas el desarrollo de dicha Reforma ha sido diferente, provocando grandes desigualdades en las prestaciones de los servicios de salud mental.

El proceso de las transferencias sanitarias desde el Estado a las CCAA se ha realizado en los últimos años, concluyendo este proceso con la aprobación de la ley 21/2001 de 27 de Diciembre (2). Esta Ley regula las medidas fiscales y administrativas del nuevo sistema de financiación en materia de sanidad.

Desde enero del año 2002 la gestión de la salud y, por ende de la salud mental, es responsabilidad de las Consejerías de Salud o Sanidad de cada CCAA. A partir de ahí fue necesario la transformación del Sistema Nacional de Salud y el impulso del Consejo Interterritorial como órgano de coordinación entre las CCAA y el Estado. Para regular esta coordinación se estableció un marco legal mediante la aprobación de la Ley de Cohesión y Calidad del Sistema Nacional de Salud (3). Esta Ley "establece acciones de coordinación y cooperación de las administraciones públicas sanitarias como medio para asegurar a los ciudadanos el derecho a la protección de la salud, con el objetivo común de garantizar la equidad, la calidad y la participación social en el Sistema Nacional de Salud".

La Ley además propone unos instrumentos para la toma de decisiones que corresponderán asumir conjuntamente al Estado y Comunidades Autónomas. Dichos instrumentos los cataloga como Órganos Especializados e incluye entre ellos el Observatorio del Sistema Nacional de Salud. Define éste como órgano dependiente del Ministerio de Sanidad y Consumo que proporcionará un "análisis permanente del Sistema Nacional de la Salud en su conjunto mediante estudios comparados de los servicios de salud de las comunidades autónomas en el ámbito de la organización, provisión de servicios, gestión sanitaria y resultados". (Cap. VI: de la Calidad, sección 2, Art. 63). 
El Observatorio se creó el 29 de agosto de 2003 (RD 1087/2003, de 29 de agosto). Es un órgano dependiente de la Agencia de Calidad del Ministerio de Sanidad y Consumo.

Paralelamente, la Asociación Española de Neuropsiquiatría (AEN) valoró la necesidad de promover la creación de un observatorio específico de salud mental e impulsó su puesta en marcha con la participación de las Asociaciones Autonómicas.

La AEN planteó como objetivo de dicho Observatorio ofrecer "un análisis permanente del sistema de Salud Mental facilitando información objetiva, fiable y comparable de las diferentes CCAA".

\section{Metodología}

Para la instauración del observatorio de salud mental se ha organizado un Grupo de Trabajo constituido con la participación de todas las Asociaciones Autonómicas integradas en la AEN. Los miembros constituyentes de este grupo han sido nombrados por la Juntas Directivas de cada Asociación Autonómica. Cada uno de ellos es el responsable / representante de su Autonómica en el observatorio de salud mental (tabla 1)de la AEN.

Tabla 1. Responsables del observatorio

\begin{tabular}{|l|l|}
\hline COMUNIDADES AUTÓNOMAS & RESPONSABLES \\
\hline ANDALUCIAA & Fermín Pérez \\
\hline ARAGÓN & Álvaro Monzón \\
\hline ASTURIAS & Cesar Sanz de la Garza \\
\hline BALEARES & Isabel Salvador, Laura de la Fuente \\
\hline CANARIAS & Cecilio Hernández de Sotomayor \\
\hline CANTABRIA & Oscar Vallina \\
\hline CASTILLA LA MANCHA & Maria del Mar Soler \\
\hline CASTILLA LEÓN & Pilar de la Viña \\
\hline CATALUNAA & Belén Díaz Mújica, Cristina Gisbert \\
\hline EUSKADI y NAVARRA & Ander Retolaza \\
\hline EXTREMADURA & Margarita Silvestre García \\
\hline GALICIA & Fernando Márquez Gallego \\
\hline MADRID & Iván de la Mata \\
\hline MELILLA & Joaquín López de Guevara, Rosa Clarés \\
\hline MURCIA & Marisa Terradillos \\
\hline RIOJA & Carmen Merina Díaz \\
\hline VALENCIA & Manuel Gómez Beneyto, Mikel Munárriz \\
\hline
\end{tabular}

En concreto, los pasos realizados han sido:

1. Análisis de los datos que el cuestionario elaborado por la anterior Junta Directiva de la AEN había a portado a fecha de Diciembre del 2002.

2. Reelaboración del Cuestionario (Anexo I). 
3. Cumplimentación por parte del responsable del Cuestionario actual con los datos sobre la atención a salud mental en su CCAA a fecha de Diciembre del 2003.

4. Recogida e informatización de todos los datos para realizar su análisis.

En este Informe presentamos los datos aportados por los diferentes responsables de la AEN en cada CCAA, actualizados a Diciembre del 2003. Así mismo se ha realizado un análisis comparativo, cualitativa y cuantitativamente de la situación de la atención a la Salud Mental de las Comunidades Autónomas y de la Ciudad Autónoma de Melilla. 